\title{
CUTTING PROCESS SIMULATION ON THE BASIS OF RHEOLOGICAL PROPERTIES OF METALS
}

\author{
Jüri Olt ${ }^{\mathrm{a}}$, Viacheslav V. Maksarov ${ }^{\mathrm{b}}$ \\ ${ }^{a}$ Institute of Technology, Estonian University of Life Sciences, 56 Kreutzwaldi Str., EE51014, Tartu, Estonia \\ ${ }^{b}$ Department of Mechanical Engineering, National Mineral Resources University, 21 Line, Vasilevsky Island, St. \\ Petersburg, Russia
}

\begin{abstract}
The aim of the research was chip formation simulation in the process of metal cutting on the basis of rheological properties of metals. Complex rheological models have been analyzed, permitting the author to make an assumption and substantiation for forming the chip formation process hypothesis. Research has shown that the rheological model in the form of series connection of the Ishlinsky elastoviscoplastic relaxation medium (reflecting the process of initial deformation of the metal layer being cut off) and the Voigt medium with two elastic-dissipative elements (reflecting the process of deformation and friction of the chips being removed) reflects the chip formation process with regard to plastic deformation and fracture of metal in a local zone most completely. The obtained complex rheological model was the basis for the development of an adequate dynamic model of the chip formation process.
\end{abstract}

Keywords: metal cutting, modelling, chip formation, rheological models, metal deformation.
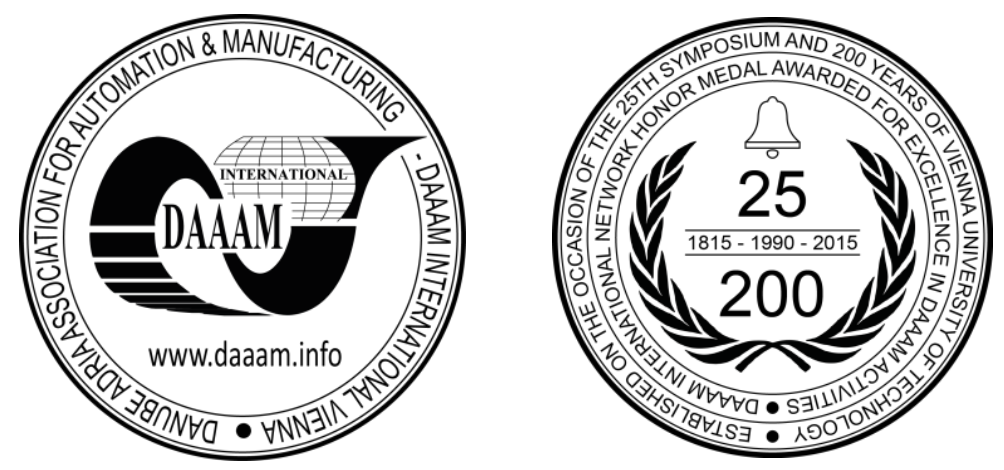

This Publication has to be referred as: Olt, J[ueri] \& Maksarov, V[iacheslav] (2016). Cutting Process Simulation on the Basis of Rheological Properties of Metals, Proceedings of the 26th DAAAM International Symposium, pp.02290237, B. Katalinic (Ed.), Published by DAAAM International, ISBN 978-3-902734-07-5, ISSN 1726-9679, Vienna, Austria

DOI:10.2507/26th.daaam.proceedings.032 


\section{Introduction}

In order to control the cutting process, one must determine the physical nature of the phenomena occurring in the cutting zone, their impact on the cutting process stability, and determine the conditions for its stabilisation. Among the cutting process perturbing factors, the determinate and the random ones can be elicited. Regular changes of cutting speed and depth, the tool geometry caused by the design features of the workpieces being machined, and the cutting kinematics can be classified as the determinate perturbing factors. The random perturbing factors are as follows: local oscillations of the mechanical properties of the material being machined, tolerance oscillation (determined by the workpieces manufacture method), and irregularity of the workpiece (tool) rotation or the tool motion (advance) [1, 5, 7, 9].

Metal cutting is a complex physical and mechanical process including plastic deformation and fracture of metal, friction in the process of the tool contact interaction with the material being machined; moreover, it take place under extreme conditions that have specific features not characteristic of other technical processes [4, 13]. The physical conditions of the cutting process are characterized by significantly higher parameter values than many other mechanical processes accompanied by plastic deformation $[2,3,10]$.

The basis for the machining process is the process of chip formation, i.e. separation of the layer of material being cut off from the workpiece $[6,11,17]$. The penetration of the tool cutting wedge is accompanied by compression of the material being machined. The material's resistance to compression is determined by its physical and mechanical properties (strength, hardness). It will be in direct proportion to the area of the metal layer being cut off and the instrument penetration velocity. When the material's effective stress in its interface with the cutting edge become critical, the layer being cut off begins to separate itself from the workpiece material and travels along the tool front surface.

The chip formation process is accompanied by a very significant relative deformation of material, where the deformation rate in a relatively small zone is high. That is why the cutting process can be studied on the base of idealised physical models with the help of mathematical analysis $[1,6,8,16,18]$.

The structural pattern characteristic of the cutting process includes input and output parameters. The input (primary) parameters are set by the designer (the material, the finished piece dimensions and tolerances) and the technologist (the machine, attachment, tool, process environment, and cutting conditions). The secondary (output) parameters determining the machining results include performance parameters of the finished piece such as accuracy and surface finish, durability and strength of the tool, productivity and efficiency of machining.

The theoretical and experimental research performed in the sphere of metal machining in recent years by cutting and based on dislocation visualization of the material structure permitted us to obtain a better understanding of many physical phenomena with their reference to each other. This fostered improvements in metal machining technologies in a big way.

\section{Materials and Methods}

\begin{tabular}{|ll|}
\hline Nomenclature \\
$E$ & elasticity modulus \\
$G$ & shear modulus \\
$T_{p}$ & relaxation time \\
$a$ & sheared layer thickness \\
$a_{1}$ & chip thickness to the ridge \\
$a_{2}$ & chip thickness to the cavity \\
$m_{p}$ & distance between large external chip elements \\
$v_{s}$ & cutting velocity direction \\
$\Delta x$ & deformation displacement thickness \\
$\Delta y$ & displacement of the principal periodical structure \\
$\beta_{1}$ & localized bands displacement angle \\
$\gamma$ & front angle \\
$\varepsilon$ & deformation \\
$\mu$ & friction coefficient \\
$\sigma_{H}$ & direct stress in the elastic element \\
$\sigma_{N}$ & direct stress in the viscous element \\
$\sigma_{p l}$ & plasticity limit \\
$\tau$ & tangent stress \\
\hline
\end{tabular}


According to the research results [5,8], the interaction between the cutting wedge and the work material in the process of flat chip formation was studied before the moment of chip formation in two stages: deformation up to the shearing plane and the drift of a shallow element along the shearing plane.

As a result of those processes, a fine AP type platelet structure is created in the layer being sheared. It consists of wide platelets and narrow shear planes of uniform cyclicity and practically invariant to the cutting conditions (Figure 1), i.e. a fine periodic basic structure is formed. It only reflects the properties inherent to the work material.

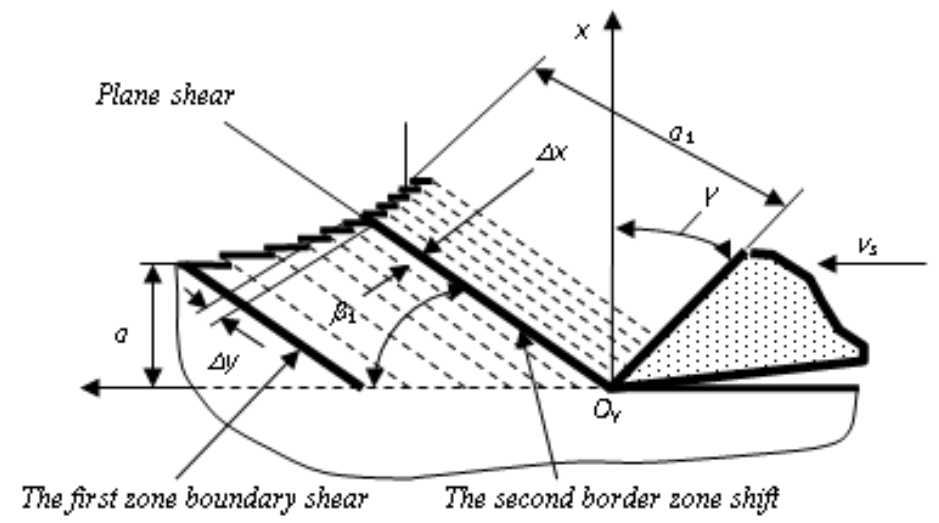

Fig. 1. Conventional model of localized shear bands in the primary plastic deformation zone of the layer being sheared.

A chip's descent along the front surface of a tool is determined by relationships between the tangent stresses $\tau$ and the direct ones $\sigma$ at each contact point, determining the local chip against the front surface friction coefficient $\mu$. The friction coefficient value is at its greatest at the chip separation point, and gradually reduces on drawing near the tool cutting edge.

Tangent stresses may only increase in a limited way; therefore, when external friction stress exceeds the chip material resistance to flow shear, external friction exceeds the chip material resistance to flow shear, and external friction is replaced by internal shears in the chip material that are more acceptable energetically. The chip travels because of the plastic deformations in its contact layer. Whereupon the lower layer of the chip reduces the travel velocity along the front surface, the aggregate result being the formation of large saw-toothed elements $B_{P}($ Figure 2)

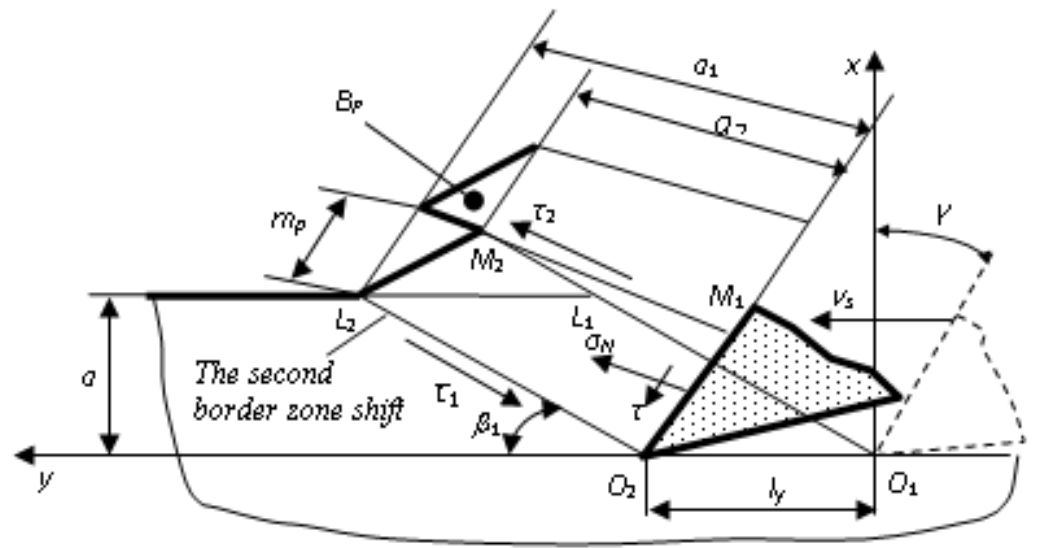

Fig. 2. Conventional model of chip making process.

The physical properties of the metal being deformed in the process of edge cutting machining can be reflected with the help of discrete mechanical (rheological) models. By rheological phenomena, we mean the phenomena of materials deformation alteration in the course of time $[12,14,15]$. Discrete rheological models visualize the pattern of change in material deformation and stresses depending on its structure that determines the material properties under mechanical stress.

Material stress strain behaviour in the plasticity zone during cutting. A complex physical and mechanical process of metal plastic deformation and fracture takes place at the interface between the cutting instrument and the work material, causing chip formation and its separation from the workpiece. As we consider the interaction between the cutting wedge and the work material, we may simplify the motion analysis by visualizing the deformed area of the chip layer being sheared as a continuous medium (a material continuum).

A phenomenological approach is used for mathematical decryption of the properties of this area of the metal layer being sheared, whereupon regular patterns found as a result of experiments and newly introduced hypotheses are 
used. Continuous media mechanics analyses movements of and interactions between volume elements, rather than atoms. A plasticity theory boundary problem was considered in order to develop a closed set of equations simulating the thermoelastic-plastic process of chip formation.

\section{Rheological models}

The physical properties of metal being deformed can be visualized as a mechanical analogue (rheological model) being a totality of mechanical elements: elasticity, viscosity, and plasticity [14, 15]. Each of them or their combination characterizes the basic (fundamental) properties of the material and permits one to visualize the stressstrain behaviour of metal under the impact of external loads. All other mechanical properties are derivatives of the fundamental constants - fixed factors that cannot be calculated on the basis of other characteristics of the present structural level.

Rheological models (the phenomena of deformation alteration over time are known as rheological ones) visualize the nature of changes in material stresses and deformation depending on its structure determining its properties under mechanical impact. An elementary model of elastic material is visualized as elastic elements having appropriate properties. The relationship between stress and deformation under load and upon load removal is linear. It is determined by the well-known Hooke's law: $\sigma=E \cdot \varepsilon$ (wherein $\sigma$ is stress, $E$ is the elasticity modulus, $\varepsilon$ is deformation.

The elementary model of an ideal plastic or rigid-plastic material is visualized as a dry friction element (the Saint Venant model). Under external load, a material like this does not deform until the stress exceeds a certain plasticity limit $\left(\sigma_{p l}\right)$. The trigger event of plastic deformation as a residual shearing deformation is determined by the relationship: $\sigma=\sigma_{p l}$. Hardened plastic material is conventionally visualized as a set of elementary models connected in series. Each of the models is put into operation as shear advances.

The viscous body model is visualized as a linear damper whose resistance is proportional to the elements' relative motion velocity (the Newton model). The work of external forces against viscous resistance forces is irrevocable. The stress in the viscous model is proportionate to the load application velocity: $\sigma_{N}=2 \eta \cdot \dot{\varepsilon}_{N}$, wherein $2 \eta$ is the resistance coefficient.

Complex rheological models as a mechanical combination of simple models permit one to describe the properties of real materials (with the necessary approximation). They take shape when elementary rheological models are connected in series and in parallel.

The general rheological equation used to consider various rheological models can be written down as follows:

$\sigma-\sigma_{m}=G \cdot\left(\varepsilon+T_{p} \cdot \dot{\varepsilon}\right)-T_{p} \cdot \sigma$

wherein $\sigma$ is stress, $\sigma_{m}$ is yield point, $\varepsilon$ is (shear) deformation, $\dot{\varepsilon}$ is deformation velocity, relaxation time $T_{p}=\eta / G$.

Three basic rheological models were considered in order to describe the cutting process mathematically with regard to metal fracture in the chip making zone (Figure 3) [9, 12]: viscoelastic Maxwell model $\boldsymbol{M}$, visco-hereditary Voigt model $\boldsymbol{F}$, and elastoplastic Prandtl model $\boldsymbol{P}$. In all models, $H$ and $N$ elements reflect elastic and damping proprieties, while the plasticity limit $\sigma_{p l}$ is imitated by the Saint Venant model (St.V).

It is of interest to consider the behaviour of models in three cases: 1) $\varepsilon=$ const, 2) $\sigma=$ const and 3) $\sigma(t)=\sigma_{0} \cdot \sin \omega t$ or $\varepsilon(t)=\varepsilon_{0} \cdot \sin \omega t$, when stresses $\sigma(t)$ or deformations $\varepsilon(t)$ in a rheological body change harmonically. In all the above cases, relaxation processes take place in the bodies being deformed, i.e. in the first case it is relaxation of stresses, while in the second one, it is creep processes

Relaxation of stresses is a phenomenon observed in the majority of actual materials; it is essentially gradual reduction (over time) of the stresses in a body at a set deformation value.

The Voigt equation takes the following form (Figure 3)

$$
\sigma(t)=2 \mu \cdot \varepsilon(t)+2 \eta \cdot \dot{\varepsilon}(t)
$$

The solution of this equation at any function $\sigma(t)$ and at initial conditions $\varepsilon(0)=\varepsilon_{0}$ takes the following form: .

$$
\sigma(t)=\exp \left(-\frac{\mu}{\eta} \cdot t\right) \cdot\left[\varepsilon_{0}+1 / 2 \eta \int_{0}^{t} \sigma(\tau) \exp \left(\frac{\mu}{\eta}\right) d \tau\right]
$$

The analysis of equation (11) at $\varepsilon=$ const demonstrates that the Voigt rheological body behaves like the Hooke elastic medium, because at $\dot{\varepsilon}=0$, the viscous element does not take load and the Voigt model does not describe the relaxation phenomenon. 
At $\sigma=\sigma_{0}=$ const, we obtain the following equation:

$$
\varepsilon(t)=\frac{\sigma_{0}}{2 \mu}+C \cdot \exp \left(-\frac{\mu}{\eta} \cdot t\right)
$$

wherein $C$ is the integration constant determined on the basis of the initial conditions. In case $\varepsilon(0)=0$, the value is $C=-\frac{\sigma_{0}}{2 \mu}$; then as time period $t$ increases, the $\exp \left(-\frac{\mu}{\eta} \cdot t\right)$ member decreases rapidly and at $t \rightarrow \infty$ deformation tends to its balance value $\varepsilon(\infty)=\frac{\sigma_{o}}{2 \cdot \mu}$.

a)

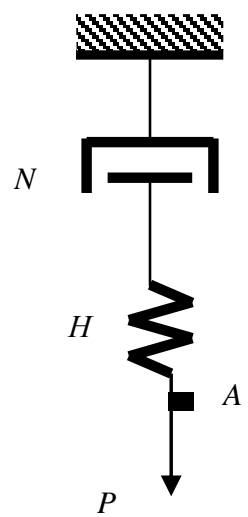

$P=H-N$ b)

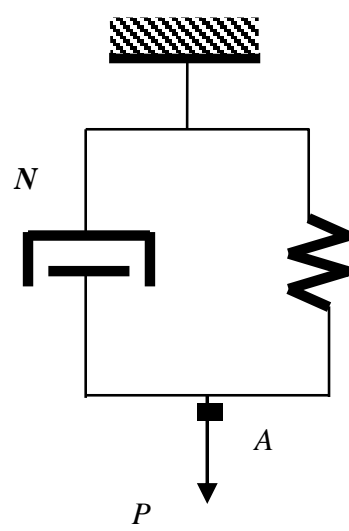

$P=H / N$ c)

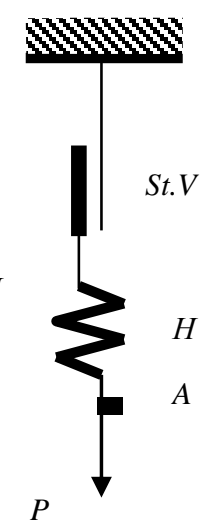

$P=H-S t . V$

Fig. 3. Mechanical interpretation of rheological bodies with two-element schemes:

a) Viscoelastic Maxwell Model; b) Visco-Hereditary Voigt Model; c) Elastoplastic Prandtl model.

Under constant loads, the deformation velocity value has the following form:

$$
\dot{\varepsilon}(t)=\left(\frac{\sigma_{0}}{2 \mu}\right) \cdot \exp \left(-\frac{\mu}{\eta} \cdot t\right)
$$

If the period of time during which deformation changes in the Voigt model is very short, then $\exp \left(-\frac{\mu}{\eta} \cdot t\right) \approx 1$ and equation (13) will have the following form: $\dot{\varepsilon}(t) \approx \frac{\sigma_{0}}{2 \mu}$

It follows thence that under rapid loading the Voigt model behaves like the Newton medium, while under slow loading it behaves like the Hooke ideal elastic model.

The analysis of the changes in deformation in the Voigt model for the period of time $t=t_{l}$ demonstrates that upon load removal the following deformations will occur:

$$
\varepsilon(t)=\frac{\sigma_{0}}{2 \mu} \cdot\left[1-\exp \left(-\frac{\mu}{\eta} \cdot t_{1}\right)\right] \cdot \exp \left[-\frac{\mu}{\eta} \cdot\left(t-t_{1}\right)\right]
$$

If we take the unloading moment $t=t_{1}$ as the reference point, expression (6) can be simplified:

$$
\varepsilon(t)=\varepsilon_{1} \cdot \exp \left(-\frac{\mu}{\eta} \cdot t^{\prime}\right)
$$

wherein $t^{\prime}=t-t_{1}$. It can be concluded from expression (7) that after the load is removed, deformation in the Voigt rheological body varies as a function of 
$\tau_{\text {ret }}=\frac{\eta}{\mu}$

known as the delay time. At $t^{\prime}=\tau_{r e t}$ deformation $\varepsilon(t)$ will be $\varepsilon(t)$ times less than the initial one $\varepsilon_{1}$. In case $\tau_{\text {ret }}=\tau_{r e l}$ we can conclude that the delay time is equal to the relaxation time. For more complex rheological models, $\tau_{\text {ret }} \neq \tau_{\text {rel }}$.

If the stress in the Voigt medium changes harmonically according to the formula $\sigma(t)=\sigma_{0} \cdot \sin \omega t$, equation (3) will take the following form:

$\dot{\varepsilon}(t)+\frac{\mu}{\eta} \cdot \varepsilon(t)=\frac{\sigma_{0}}{2 \eta} \cdot \sin \omega t$

then $\varepsilon(t)=A \cdot \sin \omega t+B \cdot \cos \omega t ; A=\varepsilon_{0} \cdot \cos \varphi ; B=\varepsilon_{0} \cdot \sin \varphi$ wherein $\varepsilon_{0}-$ is the amplitude and $\varphi$ is the phase difference Constants $A$ and $B$ are determined by a set of algebraic equations: $A \cdot \omega+\frac{\mu}{\eta} \cdot B=0, A \cdot \frac{\mu}{\eta}-B \cdot \omega=\frac{\sigma_{0}}{2 \eta}$

therefore

$$
A=\sigma_{0} \cdot \frac{\mu}{2}\left(\mu^{2}+\eta^{2} \cdot \omega^{2}\right), B=-\sigma_{0} \cdot \omega \cdot \frac{\eta}{2}\left(\mu^{2}+\eta^{2} \cdot \omega^{2}\right)
$$

then

$$
\varepsilon_{0}=\frac{\sigma_{o}}{2 \cdot \sqrt{\left(\mu^{2}+\eta^{2} \omega^{2}\right)}}, \tan \varphi=-\omega \cdot \frac{\eta}{\mu}
$$

Frequency (or relaxation time $\tau_{\text {rel }}=\eta / \mu$ ) variations from zero to infinity change $\varepsilon_{0}$ amplitude from $\sigma_{0} / 2 \eta$ to zero (i.e., deformation fluctuations are damped), and phase shift $\varphi$ surges from zero to $-\pi / 2$. Thereby, deformation variations in the Voigt model are delayed as compared to the stress variation

Thus, analysis of the behaviour of Maxwell and Voigt rheological models has demonstrated that relaxation processes occur in both of the above models, but only in the Voigt model a delay in the process can be observed after load removal. This is a characteristic feature according to phenomenological visualisation of the cutting process with regard to plastic deformation and metal fracture in the chip formation zone.

Complex rheological models. Rheological models with three- and four element aperiodical patterns are of the greatest interest for cutting simulation. Joining the Hook, Newton and St. Venant single-element schemes, as well as Maxwell, Voigt and Prandtl two-element schemes in a complex model permits us to obtain new properties reflecting the process of solid body deformation and fracture taking place during metal cutting most completely. The complex rheological bodies that are most acceptable for visualisation of the phenomena occurring in the first plastic zone of deformation of the layer being sheared are shown in Figure 4. Among such elements are the three-element Bingham rheological bodies and the Ishlinsky $(S C H W)$ and Shvedov $(J)$ four-element rheological bodies. Plastic deformation in complex models is reflected by element $H$ (Figure 4, $a$ ) and $H_{1}$ (Figure 4, $b, c$ ), plastic deformation, by $H_{2}$, damping, by $N$, while attainment of the plasticity limit $\sigma_{p l}$ is imitated by the St. Venant element (St.V.).

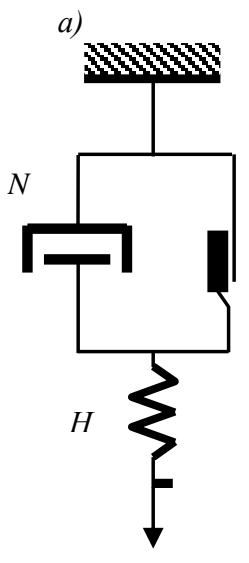

$B=H-N / S t . V$

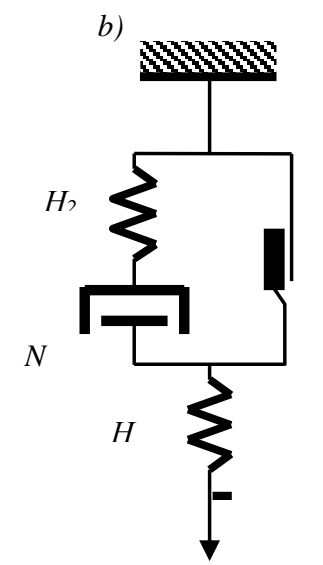

$$
S C H W=(M / S t . V)-H_{l}=
$$$$
=\Gamma\left(H_{2}-N\right) / S t . V I-H_{1}
$$

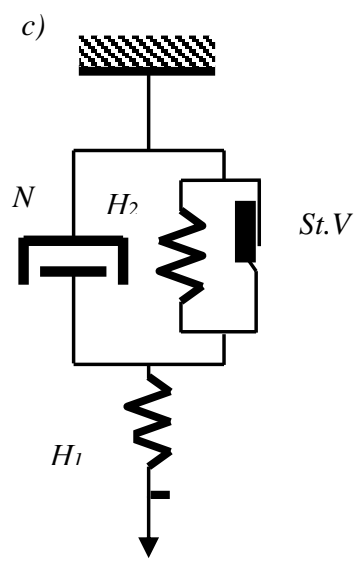

$J=\left[N /\left(H_{2} / S t . V\right)\right]-H_{l}$

Fig. 4. Mechanical interpretation of complex rheological models:

a) Bingham Rheological Body; b) Shvedov Rheological Body; c) Ishlinsky Rheological Body. 
Various combinations of the elastic deformation elements, as well as combination of viscous deformation with the elements of damping or plasticity permit us to visualise the mechanical interpretation of the chip making process.

The model of the Bingham rheological body is shown in Figure 4, $a$. When the strength limit of element $H \sigma_{\mathrm{N}}<\sigma_{\mathrm{pl}}$ is reached, wherein $\sigma_{p l}$ is the ultimate loading of a plastic element, the Bingham medium behaves like the Hooke's elastic medium. At equal or greater stresses, it demonstrates the properties of the Maxwell medium, since according to the terms $\sigma_{p l}=$ const in a plastic element. The equation of the Bingham rheological body can be written down as follows:

$$
\begin{aligned}
& \text { for }\left|\sigma_{H}\right|<\left|\sigma_{p l}\right| ; \\
& \sigma=2 \mu_{H} \cdot \varepsilon,\left|\sigma_{H}\right|=\left|\sigma_{p l}\right| \\
& \text { for }\left|\sigma_{H}\right| \geq\left|\sigma_{p l}\right|, . \\
& \sigma-\sigma_{p l}=2 \eta_{N} \cdot \dot{\varepsilon}_{N}, \frac{\dot{\sigma}}{2 \mu}+\frac{\sigma-\sigma_{p l}}{2 \eta}=\dot{\varepsilon}
\end{aligned}
$$

The model of the Shvedov rheological body is shown in Figure 4, b. If we presume that the rigidity factor of the elastic element $\mathrm{H}_{2}$ in the Shvedov model is equal to infinity, then the Shvedov medium is transformed into the Bingham medium

The mechanical model of the elastoviscoplastic Ishlinsky medium is shown in Figure 4, c. According to this diagram, at $\left|\sigma_{p l}\right|>\left|\sigma_{H}\right|$,

$$
\sigma(t)=2 \mu_{1} \cdot \varepsilon(t)
$$

i.e. at those conditions, the Ishlinsky rheological body is the Hooke model.

At $|\sigma|>\sigma_{p l}$ such a rheological body behaves like a viscoelastic medium. When we exclude values $\varepsilon_{1}, \varepsilon_{2}, \varepsilon_{3}$ from the relationships

$$
\begin{aligned}
& \varepsilon=\varepsilon_{1}+\varepsilon_{2}, \quad \varepsilon_{2}=\varepsilon_{3}, \sigma=2 \mu_{1} \cdot \varepsilon_{1} \\
& \sigma \pm \sigma_{p l}=2 \eta \cdot \dot{\varepsilon}_{3}+2 \mu_{2} \cdot \varepsilon_{2}
\end{aligned}
$$

we get a rheological equation for $|\sigma|>\left|\sigma_{p l}\right|$ that looks like this:

$$
\dot{\sigma}(t)+\frac{\mu_{1}+\mu_{2}}{\eta} \cdot \sigma(t)=2 \mu_{1} \cdot \dot{\varepsilon}(t)+\frac{2 \mu_{1} \cdot \mu_{2}}{\eta} \cdot \varepsilon(t) \pm \sigma_{p l} \cdot \frac{\mu_{1}}{\eta}
$$

Presuming that $\sigma \mathrm{p}=0$ in equation (2), we shall have the Kelvin medium equation.

A force applied to point $A$ of the model under consideration will first deform only the external elastic element; this is characterized by the parameter $\mu_{1}$. The elastic behaviour of the Ishlinsky rheological medium corresponds to this state.

\section{Discussion}

At $\left|\sigma_{l}\right|>\left|\sigma_{p l}\right|$, a relative displacement occurs in the viscous element and the internal elastic element is deformed at the same time. It is evident that stresses $\sigma_{2}=2 \mu_{2} \cdot \varepsilon_{2}$ and $\sigma_{3}=2 \eta \cdot \dot{\varepsilon}_{3}$ arise, whereupon the condition $\varepsilon_{2}=\varepsilon_{3}$ is retained. Under prolonged impact of stress $\left|\sigma_{l}\right|>\left|\sigma_{p l}\right|$, the position of the viscous element will change. After a sufficiently long time period, the relative position of the viscous element will be determined by the deformation of the internal elastic element. When the load is quickly removed, the external elastic element tracks the viscous element directly and passes into its 'natural' stands virtually instantaneously.

At $\sigma_{2}<\sigma_{p l}$, deformation of the plastic element ceases at a position characterized by residual deformation. The viscous element is fixed in the respective position. Since the viscous element has no internal strains $\left(\dot{\varepsilon}_{3}=0\right)$ at the moment when the plastic element stops, the internal elastic elements deform when $\sigma_{2}<\sigma_{n n}$. This state of the model can be taken as an 'initial' one that does not correspond to its 'natural' state. Should we change the direction of external forces, the force needed to shear the plastic medium as a consequence of residual strains $\sigma_{2}$ in the internal elastic 
elements will be less than the $\sigma_{p l}$ being determined (in absolute values) and at $\sigma=\sigma_{2}$ the model will start shifting. Thus, the Ishlinsky model describes the effect caused by reduction of the plastic limit under repeated load by a force acting in the opposite direction before the plastic limit is reached (the Bauschinger effect). The model makes it possible to put together the processes of brittle and ductile material fracture if we assume that the brittle fracture corresponds to the fracture of the external elastic element and the ductile one to the fracture of the internal elastic element. Actually, when $\sigma>\sigma_{p l}$ tension arises quickly in the model, the viscous element $\dot{\varepsilon}_{3}$ velocity will be very significant and therefore significant forces will arise in it. The behaviour of all other elements in this model at $\dot{\varepsilon}_{3} \rightarrow \infty$ will be characterized by the viscous element behaviour. The deformation of the whole model will be mainly characterized by the deformation of the external elastic element since the viscous element cannot shift to a noticeable distance in a very short period of time. This is a suitable method for brittle fracture modelling.

Should we assume that the rigidity coefficient of the internal elastic element in the model is smaller than that of the external elastic element, under a rapid or sufficiently slow loading the model with stresses $\sigma>\sigma_{p l}$, the internal elastic element will be fractured after some time. The fracture of the internal elastic element is preceded by relative displacement of the viscous element accompanied by displacement of the plastic element. This corresponds to the increase in the 'plastic deformation' of the internal elastic element, causing an abatement of forces in the external elastic element. In terms of models, this corresponds to relaxation of forces in the actual bodies being deformed.

The analysis of rheological schemes permits us to conclude that as the number of elastic, viscous, and plastic elements in mechanical models increases, a model never goes beyond the elementary linear deformation law, since the rheological equations for all such models will be ordinary linear differential equations with constant factors (since the respective rheological parameters $\mu, \eta$ and $\sigma_{p l}$ of the elastic, viscous, and plastic elements are constants). Figure 4 demonstrates that the Bingham medium is a particular case of the Shvedov medium, since the rigidity factor of elastic elements $\mathrm{H}_{2}$ (being a part of the Maxwell model) is equal to infinity. The Ishlinsky model also transforms itself into the Bingham model on the same condition, i.e. the rigidity factor of element $H_{2}$ approaches $\infty$. It should be noted that at $|\sigma|$ $>\left|\sigma_{p l}\right|$ the rigidity coefficient of element $\mathrm{H}_{2}$ becomes equal to zero, and the Ishlinsky model describes the Bingham model.

Thereby, the Ishlinsky four-element rheological model can best reflect the dynamics of plastic deformation and fracture of solid body in the layer of metal being sheared in case of modelling chip making curing which stresses exceeding yield point $\sigma_{p l}$ arise and viscous element $N$ modelling diffusion relaxation processes connected in parallel to element $\mathrm{H}_{2}$ describing strain hardening.

\section{Conclusion}

As a result of the analysis performed, we found that the chip formation process is accompanied by spatial inhomogeneity of plastic deformation of metal in the metal layer being sheared.

We have put forward and substantiated a hypothesis describing the chip formation process. It presumes that a fine platelet type structure is created in the primary plastic deformation zone of the layer being sheared with uniform periodicity reflecting only the work material properties and insensitive to changes in cutting conditions. In the secondary plastic deformation zone where chips contacting with the cutting tool front surface are subjected to secondary deformation under the impact of friction forces, large external chip elements (sawtooth structures) are formed.

A rheological model has been obtained in order to analyse the cutting process with regard to plastic deformation in the local chip-forming zone. This model has the form of series connection of the Ishlinsky elastoviscoplastic relaxation medium (reflecting the process of initial deformation of the layer of metal being cut off) and the Voigt medium with two elastic-dissipative elements (reflecting the process of deformation and friction of the chips being removed).

\section{References}

[1] Atkins, A.G. Modeling metal cutting using modern ductile fracture mechanics: quantitative explanations for some longstanding problems. International Journal of Mechanical Sciences 45 (2003) 373-396.

[2] Bruce L., Bramfitt, A., Benscoter, O. Metallographer`s Guide. ASM International, 2002: pp.307-316.

[3] Engin, S., Altintas, Y. Mechanics and dynamics of general milling cutters. Part II: inserted cutters. International Journal of Machine Tools \& Manufacture 41 (2001) 2213-2231.

[4] Eugene, I., Rivin Hongling, K. Enhancement of Dynamic Stability of Cantilever Tooling Structures. International Journal of Machine Tools and Manufacture 32 (4) 1992: pp. 539-561.

[5] Maksarov, V.V., Olt, J. 2010. Influence of local metastability in the workable material on control of the cutting process. 7th International DAAAM Baltic Conference "Industrial Engineering", April 22-24, 2010, Tallinn, Estonia, 226-231.

[6] Maksarov, V.V., Olt, J., Laatsit, T., Leemet, T. Physical argumentation of deformation process potential control while cutting heavily processed material. 6th International DAAAM Baltic Conference "Industrial Engineering", April 24-26, 2008, Tallinn, Estonia. 263-268. 
[7] Olt, J., Maksarov, V.V., Madissoo, M. Dynamic stabilization of technological systems for processing edge cutting through local metastability. IEEE International Symposium on Assembly and Manufacturing, May 25-27, 2011, Tampere, Finland. 6-11.

[8] Olt, J., Maksarov, V.V. Using an anisotropic properties of sheetmetal to develop a design of vibrationless cutting tool. Agronomy Research, Biosystem Engineering, Special Issue 1 (2012) 181-186.

[9] Otto, A., Radons, G. The influence of tangential and torsional vibrations on the stability lobes in metal cutting. Nonlinear Dynamics, 82(4) (2015) 1989-2000.

[10] Panin, V.E. Structural levels of plastic deformation and destruction. Nauka/Science, Novosibirsk, 2011 , pp. 251.

[11] Radulescu, M., Radulescu, B., Cozminca, I. Self-sustained vibrations. Annals of DAAAM for 2008 \& Proceedings of the 19nd International DAAAM Symposium, pp. 1163-1164.

[12] Senecaut,Y., Watremez, M., Brocail, J., Fouilland-Paille, L., Dubar, L. Dynamic recrystallization observed at the tool/chip interface in machining. 18th International ESAFORM Conference on Material Forming, Key Engineering Materials, 651-653 (2015) 1223-1228.

[13] Tony, L., Schmitz, K., Smith, S. Machining Dynamics. Springer, New York, 2009: pp 7-98.

[14] Vladimirov, I. The physical nature of the fracture of metals. Metallurgy, Moskow, 1984, pp. 280.

[15] William D. Callister, David G. Rethwisch. Fundamentals of Materials Science and Engineering: An Integrated Approach, Publisher: Wiley, 2012.

[16] Wu D. W. Comprehensive Dynamic Cutting Force Model and Its Application to Wave-Removing Processes, Journal of Engineering for Industry 110 (2) (1988) 155-164.

[17] Zeqiri, H., Salihu, A., Bunjaku, A., Osmani, H., Qehaja, H., Zeqiri, F. Research of chip formation during the processing with turning. Annals of DAAAM for 2011 \& Proceedings of the 22nd International DAAAM Symposium, 22(1) 385-386.

[18] Zamashchikov, Y.I. Volume constancy and material continuity in metal cutting. International Journal of Machining and Machinability of Materials, 17(3-4) (2015) 233-258. 